\title{
Post-operative Pain Assessment and Progress in Analgesia Nursing
}

\author{
Yaqian $\mathbf{L i}^{*}$ \\ Nursing Class 10 of 2014, Yancheng Vocational Institute of Health Sciences, Yancheng, Jiangsu 224000, China
}

\begin{abstract}
Today, operation has become a common practice to treat a large number of diseases, but the pains caused by an operation leave dramatic effects on patients psychologically and physiologically. Hence, it is necessary to perform post-operative pain assessment and conduct necessary analgesia nursing for patients. In this research, the author, as a student, will analyze the generating mechanism for post-operative pain and the method of assessment, and give a brief summary of progress in analgesia nursing. It is expected that this brief summary can attract attention from the public and provide the feasible reference for related fields.
\end{abstract}

\author{
KEYWORDS \\ Post-operative pain \\ Analgesia nursing \\ Research progress
}

\section{Introduction}

Post-operative pain refers to a series of physiological, psychological and behavioral reactions of organism under the effect of surgical pain (tissue damage). In clinical nursing, pain has become the fifth vital sign after body temperature, pulse, breath and blood pressure, so it has received more and more attention. As discovered in a survey, about $95 \%$ of patients after operation feel worried, anxious and restless due to their fear of pain, while other patients experience palpitation and high blood pressure. Due to post-operative pain, patients are too apprehensive in breathing, too afraid to cough or move body actively, so it is difficult to dislodge secretions and it causes atelectasis and lung infection, etc. Post-operative pain is common to all patients, which was the problem that every patient must face directly after operation $[1,2]$. Clinically, it is especially important to assess the pain of patients after an operation and provide effective analgesia nursing for them. The author, as a student, will conduct the research and survey on the progress in post-operative pain assessment and analgesia nursing. The research is

Copyright $\odot 2016$ Yaqian Li

doi: $10.18686 /$ jn.v5i4.7

Received: August 27, 2016; Accepted: November 8, 2016; Published on-line: December 29, 2016

This is an open-access article distributed under the terms of the Creative Commons Attribution Unported License (http://creativecommons.org/ licenses/by-nc/4.0/), which permits unrestricted use, distribution, and reproduction in any medium, provided the original work is properly cited.

${ }^{*}$ Corresponding author: Nursing Class 10 of 2014, Yancheng Vocational Institute of Health Sciences, Yancheng, Jiangsu 224000, China. E-mail; yaqian2014@hotmail.com summarized as follows:

\section{Guidance before operation}

When the time of operation has been determined, guidance before operation can be carried out to explain and learn about the psychology of the patient about the operation. As patients have different personalities, occupations and cultural backgrounds, their psychological reactions may be affect by different factors. Thus, it is necessary to eliminate the ideological concerns of patients in a focused manner, and explain purpose, method of operation and post-operative precautions based on the disease of the patient. Especially, it is necessary to explain the category of drainage tube used after operation, together with its purpose and duration, the degree of pain will occur at the position of drainage, change of body position, traction cut, cough and other conditions causing pain, etc. to patients, and also tell them the ways to alleviate pain.

\section{Two-way information exchange}

Before operation, information regarding post-operative analgesia must be share with patients, in order to help them understand the harms of taking excess of pain killers. Moreover, if the pain becomes worse, more drugs must be needed to control pain. Thus, it encourages patients to report the condition to doctor and nurse before the pain gets worse. Also, patients after operation can be introduced to the patients who are about to receive operation, so that they can vividly explain post-operative pain and pain experienced at the time of placing and removing the drainage tube, making the latter ready for post-operative pain. 


\section{Generation mechanism of post-operative pain and its impacts on organism}

\subsection{Generation mechanism of post-operative pain}

The generating of a patient's post-operative pain can be analyzed as two aspects. The first aspect is the feeling of pain caused by the operation, ischemia and injury, etc. [3]. The second aspect is the most direct response of an individual to harmful stimulation, which is often highly emotional and represented by a series of body movements and viscerogenic effects [4]. Post-operative pain is strictly different from common physiological pain. These pain mainly from two sources, first was the mechanical pain generated by the effect of the surgical wound on nerve endings, while the other source is the post-operative pain resulted from the damage of tissue and the sensitivity of the central nervous system.

\subsection{Impacts of post-operative pain on organism}

Post-operative pain may cause visceral reaction, which is the most significant impact on the physiological function of the organization, mainly related to the increase of catecholamine in blood and the abnormal homeostasis. It is mainly represented by arrhythmia and high heart rate, and may even result in cardiac arrest and respiratory failure in severe cases [5]. This pain may cause abnormality of inflammatory mediators, worsen the primary lesion to some extent and cause ischemic lesion of the patient, inducing a series of metabolic disturbances.

\subsection{Observation of post-operative pain}

Post-operative pain is different from common physiological pain. Apart from the feeling of pain due to mechanical pain generated by the effect of the surgical wound on nerve endings, the change of sensitivity in the peripheral and central nervous systems after tissue damage is also a major cause for post-operative pain. In the meantime, post-operative pain will also cause visceral reaction, which is the largest impact on physiological function and mainly related to the abnormal automatic activity caused by pain and the increase of catecholamine in blood. It is represented by tachycardia, high blood pressure, arrhythmia, and even cardiac arrest, as well as quick and short breath. Moreover, it may also cause nausea, vomiting and sweating, etc. The abnormal release of pain-producing and inflammatory mediators will not only worsen the ischemia, hypoxia and edema of primary lesion, but also cause metabolic disorder of hormone and enzyme system in organism, by inhibit protein synthesis and accelerated decomposition, which affect the wound healing rate. Meanwhile, pain will cause the decrease of immunoglobulins and affect body immune system after operation.

\subsection{Balanced analgesia}

Better effect of post-operative pain relief cannot be achieved through only one drug, but relies on the synergis- tic of two drugs with different mechanisms. It's to improve the effect of analgesia, reduce the dose as well as adverse reactions, and balance the effect of pain relief. For instance, opium analgesics are combined with nonsteroidal antiinflammatory drugs (NSAIDs) to lower the dose of opium analgesics by $30 \% \sim 50 \%$ for the reducing in adverse reactions which caused the inhibition of breathing.

\subsection{Change of body position}

It is very common that pain is caused at a position due to long-term pressing during the operation. Comfortable lying down after an operation can alleviate pain and facilitate respiratory circulation. After operation, the body position should be adjust every two hours without causing any pain to the patient to prevent the complications of respiratory apparatuses, to promote local blood circulation and restore the peristalsis of intestines. However, this should be explained to patients in advance for their cooperation.

\section{Post-operative pain assessment}

\subsection{Factors affecting correct assessment}

The factors are mainly classified into two categories. First category was the factors that related to the patient. For instance, a patient's gender, age, educational background and personality may affect the accuracy of post-operative pain assessment to some extent. For instance, the elderly have stronger ability to withstand pain than young people, while male patients can bear more pain than female patients. Introverted people do not tell their feelings of pain to others voluntarily, and well-educated people can describe pain more accurately. Second category was the factors in connection with the medical system and nurses. Through consulting with the related references, the author discovered that the nurses in many hospitals did not understood pain sufficiently, and more than $80 \%$ of nurses did not knew the correct way to relieve pain. Moreover, post-operative pain had not been officially included into the regular rules of the medical system yet.

\subsection{Post-operative pain assessment methods}

This study mainly involves six assessment methods. The first method is the Wong-Banker faces pain scale, which mainly predicts the degree of a patient's pain based on the patient's facial expression. This method is applicable to all ages and not restricted by cultural background and gender, so the assessment is authentic and accurate. The second method is visual analogue scale, which divides a $10 \mathrm{~cm}$ scale into 10 levels with the higher levels indicating stronger feeling of pain. Before assessment with this method, it is necessary to explain the mechanism of pain and the related method of application, and the patient is required to fill in the properties of pain independently. However, this method is only applicable to conscious patients at age of above 20 . The third method is describing pain with specific 
terms, i.e. gentle, moderate, terrible, severe and unbearable. With these terms, a patient can describe the pain he is experiencing. This method can satisfy the psychological needs of patients to some extent, but it is affected by subjective factors to a large degree, so the assessment with this method is not always accurate. The fourth method is a verbal rating scale. In this method, pain is classified into five levels. For each level, descriptive words are used to describe the pain. The fifth method is a behavior anchored rating scale, which is widely applied in clinics. Assessment through this method covers screaming, crying, facial expression and verbal description, etc. The sixth method is the monitoring of change in the vital signs of a patient, mainly including detection of a patient's respiratory method and degree of blood oxygen saturation, etc. It allows the measuring of the body temperature every $15 \mathrm{~min}$ and recorded.

\section{Progress in analgesia nursing 6.1. Pre-emptive analgesia}

Pre-emptive analgesia can effectively prevent central sensitization as analgesic is used before operation to prevent agitation, which can alleviate post-operative pain to a very high degree. Nevertheless, this analgesia must follow three principles: (1) Alleviate the transmission of agitation; (2) Reduce external sensitization of patient; (3) Lower central sensitization of patient.

Pre-emptive analgesia can block the high sensitivity of the central nervous system. Especially, it is highly applicable to neuropathic pain, and achieves the effect of pain relief by changing central sensitivity. So far, pre-emptive analgesia is conducted by employing four methods: the first method is regional block; the second method is preemptive NSAID dosing; the third method is combined analgesia; and the fourth method is pre-emptive dosing of central inhibition drug. The author comprehensively analyses the current condition and believes that the research should focus on the reasonableness of drugs for pre-emptive analgesia, carry out the necessary survey on the mechanism of pre-emptive analgesia, and perform the necessary study on different degrees of pain caused by different operations.
6.2. Application of non-pharmaceutical replacement therapy and sedative analgesia

Non-pharmaceutical replacement therapy can alleviate the pain caused by operation to patients, help reduce the dose of post-operative analgesics for patient, alleviate the anxiety and pressure resulting from operation and improve the feelings of patient on the whole. This is achieved by massage and acupuncture, etc. Besides, sedative analgesia is also an important way of analgesia management. It can achieve good effect of pain relief and cause very few adverse reactions, and also it has been widely applied in clinical medicine. It mainly connects different analgesic modes with related drugs to strengthen their synthetic effect, in order to improve the effect of pain relief.

\section{Conclusion}

In this research, the author carries out a simple analysis and discussion regarding post-operative pain assessment and analgesia nursing. As a student, the author believes that it necessary to understand pain assessment and analgesia management. This is not only the research of a topic, but also the focus on effective measures to guarantee the health of patients. Hence, the author wishes that students in related majors should learn well about related operations and theories, and all medical workers should do well in their work to provide good services for patients.

\section{References}

1. Wang Y, Qu J, Yuan L. Survey on nurses' understanding of the post-operative pain of patients. Journal of Nursing Science. 2013;12(24):154-155.

2. Liu X, Zhang Y. Experience in nursing with point pressing for patients of post-operative pain. Guiding Journal of Traditional Chinese Medicine and Pharmacy. 2012;14(03):2141-2142.

3. Peng Q. Principles and implementation of postoperative pain in multiple modes. Today Nurse. 2013;04(10):1634-1634.

4. Li L, Liu X. A survey of nurses' current knowledge regarding pain. Chinese Nursing Research. 2013;24(11):254-255.

5. Zhang J. Factors affecting assessment of post-operative pain and countermeasures. Modern Nursing. 2012;14(10):251-252. 